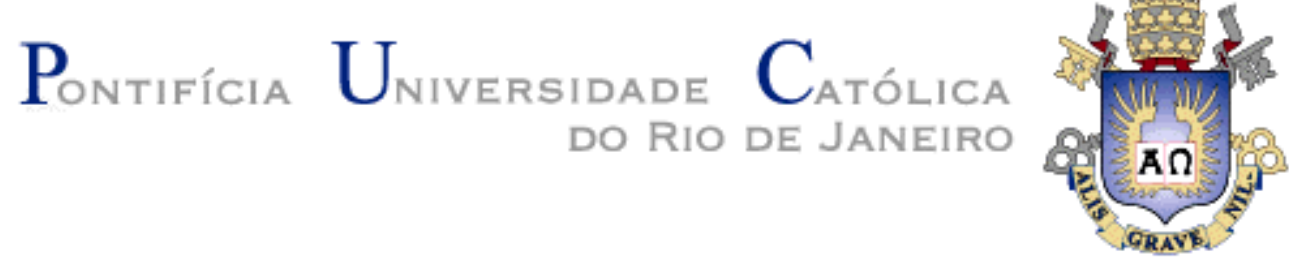

Carlos Maurício da Costa Ramos

CADEIRA DE RODAS com Design Estrutural em Tensegrity de Bambu

Tese de Doutorado

Tese apresentada ao Programa de Pós-Graduação em Design do Departamento de Artes e Design da PUC-Rio como requisito parcial para obtenção do título de Doutor em Design.

Orientador: Prof. José Luiz Mendes Ripper

Rio de Janeiro

Março de 2016 


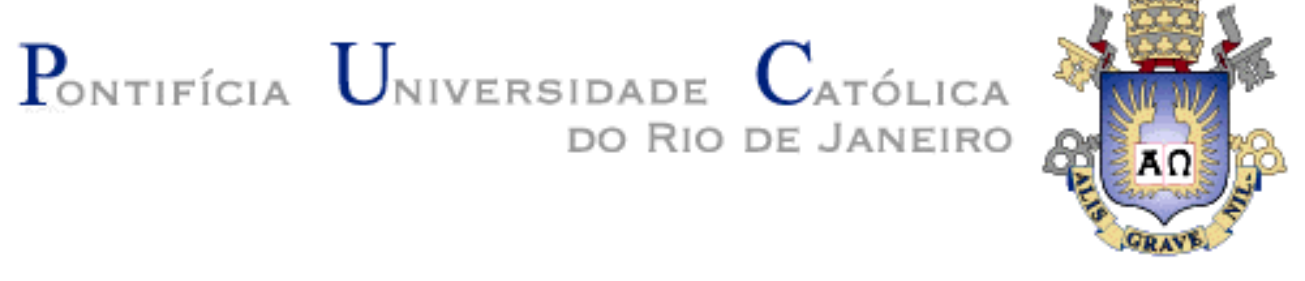

Carlos Maurício da Costa Ramos

\section{CADEIRA DE RODAS com Design Estrutural em Tensegrity de Bambu}

Tese apresentada ao Programa de Pós-Graduação em Design do Departamento de Artes e Design da PUC-Rio como requisito parcial para obtenção do título de Doutor em Design. Aprovada pela Comissão Examinadora abaixo assinada.

Prof. José Luiz Mendes Ripper Orientador - Departamento de Artes e Design - PUC-Rio

Profa. Denise Berruezo Portinari Departamento de Artes e Design - PUC-Rio

Prof. Luis Eustáquio Moreira Departamento de Engenharia de Estruturas - UFMG

Prof. Arisio Rabin Escola Superior de Desenho Industrial - UERJ

Profa. Georgia Victor Faculdade Angel Vianna-RJ

Profa. Denise Berruezo Portinari Coordenadora Setorial do Centro de Teologia e Ciências Humanas - PUC-Rio

Rio de Janeiro, 31 de março de 2016 
Todos os direitos reservados. É proibida a reprodução total ou parcial do trabalho sem autorização da universidade, do autor e do orientador.

\section{Carlos Maurício da Costa Ramos}

Graduado Desenho Industrial na habilitação de Projeto de Produto da UFRJ com projeto final "Cadeira de Rodas Ortostática", que permite ao usuário ficar na posição de pé e comercializada por dez anos e três patentes, uma de invenção, além de vários prêmios, participação em congressos e seminários em reabilitação. Pós-graduado em Marketing pela FGV-RJ e Complementação Pedagógica na Cândido Mendes-RJ e Mestre em Design na PUC-RIO em 2004. Trabalhou no Sistema FIRJAN - SESI-RJ e SENAIRJ, na Gerência de Educação a Distância como responsável pelo desenvolvimento e coordenação técnica de cursos on-line, na UNIPLI como professor universitário de Design e Arquitetura e Coordenador do curso de Design de Moda. Aprovado em 2 ํ lugar no concurso público para prof. efetivo na UFRJ em 2014 e para prof. substituto em 2015. Atualmente presta auxílio técnico no NEXT-PUC-Rio no Lab. de impressão 3D como pesquisador colaborador e prof. substituto de Laboratório de Modelos na UFRJ.

Ficha Catalográfica

Ramos, Carlos Maurício da Costa

Cadeira de rodas com design estrutural em tensegrity de bambu / Carlos Maurício da Costa Ramos; orientador: José Luiz Mendes Ripper. - Rio de Janeiro: PUC-Rio, Departamento de Letras, 2016.

v, 190 f.: il.color. ; $30 \mathrm{~cm}$

Tese (doutorado) - Pontifícia Universidade Católica do Rio de Janeiro, Departamento de Artes e Design, 2016.

Inclui referências bibliográficas

1. Artes - Teses. 2. Cadeirantes. 3. Projetos de cadeira de rodas. 4. Reabilitação. 5. Tensegrity. 6. Bambu I. Ripper, José Luiz Mendes. II. Pontifícia Universidade Católica do Rio de Janeiro. Departamento de Artes e Design. III. Título. 
Este texto diz respeito a todos que consideram a liberdade e respeito ao próximo, como meta de vida e as põe em prática ao longo do percurso.

A toda minha família que sempre acreditou e pregou todos os preceitos éticos que esmero e pauto em todas as minhas atitudes.

Em especial ao meu filho Bruno, minha esposa Raquel, meus pais Anna Amélia (in memorium), Antonio Carlos e meu irmão Fernando. 


\section{Agradecimentos}

Ao meu orientador e amigo Professor José Luiz Mendes Ripper pelo estímulo e parceria para a realização desse trabalho

A PUC-Rio, pelos auxílios concedidos, sem os quais este trabalho não poderia ter sido realizado.

Aos meus professores que tanto ajudaram a estruturar, organizar e desenvolver este texto e aos funcionários da PUC-Rio, em especial ao Romário e Diego que sempre foram extremamente profissionais e solícitos.

A todos que de alguma forma contribuíram para o sucesso desse trabalho como meus colegas de turma, a profa. Carmem Lúcia revisora do texto, aos envolvidos no desenvolvimento e produção da Cadeira de Rodas Ortostática, que deu início a todas as pesquisas na área de acessibilidade como os técnicos da UFRJ, CVI, ABBR e HU da UFRJ e logístico da Sportotec Ltda, pelo seu proprietário Raniero Bassi e a Escola de Belas Artes da UFRJ, por ceder o espaço das oficinas de metal/madeira para produção dos modelos de teste, ao professor Jose Benito Sanchez Gonzalez e os técnicos, Luis, Leandro e Gilvam.

Em especial a minha mãe Anna Amelia, falecida em janeiro de 2015, por ser a pessoa que mais me incentivou em todas as ocasiões da minha vida e teria o maior orgulho em ver seu filho conquistar mais essa importante etapa.

Estaremos sempre juntos! 


\section{Resumo}

Ramos, Carlos Mauricio da Costa; Ripper, José Luiz Mendes. "Cadeira de Rodas com Design Estrutural em Tensegrity de Bambu". Rio de Janeiro, 2016. 190 p. Tese de Doutorado Departamento de Artes e Design, Pontifícia Universidade Católica do Rio de Janeiro.

Esta pesquisa está inserida no universo dos portadores de necessidades especiais e pretende desenvolver uma nova tecnologia para projetar a estrutura de cadeira de rodas. Tal estrutura será composta por um módulo tensegrity composto de varas de bambu e cabos tensionados e adota todos os padrões antropométricos com a observância aos estudos ergonômicos e necessidades individuais de uso dos cadeirantes. Esse novo conceito de design de estrutura, por ser inédito, resulta num produto inovador e permite novas interpretações e desdobramentos. Essa pesquisa também inicia uma nova interpretação do tensegrity por sua aplicação a órteses para reabilitação que atualmente é amplamente difundido nas artes plásticas, arquitetura e no design de produto. O seu emprego pode suprir algumas necessidades como adaptação corporal favorável pela maleabilidade da estrutura que absorve os movimentos mais bruscos que possam causar incômodos, lesões e má acomodação do usuário à cadeira de rodas.

\section{Palavras-chave}

Cadeirantes; projetos de cadeira de rodas; reabilitação; tensegrity; tecnologia sustentável; bambu. 


\section{Abstract}

Ramos, Carlos Mauricio da Costa; Ripper, José Luiz Mendes. (Advisor) "Wheelchair with Structural Design in Tensegrity Bamboo". Rio de Janeiro, 2016. 190 p. PhD Thesis - Departamento de Artes e Design, Pontifícia Universidade Católica do Rio de Janeiro.

This research is part of the universe of people with special needs and aims to develop a new technology to design the wheelchair frame. This structure will consist of a compound tensegrity module bamboo poles and tensioned cables and adopts all anthropometric standards with respect to ergonomic studies and individual needs of use of the wheelchair. This new concept of structure design, to be unheard of, resulting in an innovative product and allows new interpretations and developments. This research also initiates a new interpretation of tensegrity by its application to orthoses for rehabilitation that is currently widespread in art, architecture and product design. Your job can fill some needs as body adaptation favor the flexibility of the structure that absorbs the most sudden movements that may cause nuisance, injuries and poor user accommodation to the wheelchair.

\section{Keywords}

Disable; wheelchairs projects; rehabilitation; tensegrity; sustainable tecnology; bamboo. 


\section{Sumário}

1 Introdução $\quad 22$

2 Análise ergonômica e de usabilidade em cadeira de rodas 30

2.1 Aspectos gerais e históricos da cadeira de rodas no Brasil 30

2.2 Ergonomia e usabilidade na cadeira de rodas 32

2.2.1 A importância da prescrição médica 35

$\begin{array}{ll}\text { 2.2.2 Descrição do estudo do CVI } & 37\end{array}$

2.2.3 Estudo comparativo entre o método do CVI com outros métodos conhecidos

52

3 Aplicações e aspectos formais do tensegrity 56

3.1 Aspectos e tipos formais 56

3.2 Aplicações do tensegrity 66

4 Design da cadeira de rodas tensegrity para testes de uso 80

4.1 Design do protótipo da cadeira de rodas tensegrity 80

4.2 Definição dos materiais 95

4.2.1 Vantagens na escolha e utilização do bambu 98

4.2.2 Espécie do bambu utilizado e técnicas empregadas 101

5 Produção dos modelos físicos para testes de uso 108

5.1 Metodologia empregada na produção dos modelos físicos 108

5.2 Produção do gabarito da cadeira de rodas tensegrity 109

5.3 Produção dos acabamentos metálicos e montagem dos bambus 119

5.4 Cabeamento e finalização do modelo de testes da

cadeira de rodas tensegrity

6 Estrutura, aplicação e análise dos testes com usuários

na cadeira de rodas tensegrity

6.1 Metodologia da aplicação dos testes práticos 128 
6.2 Objetivo da aplicação dos testes 132

6.3 Amostra dos testes 133

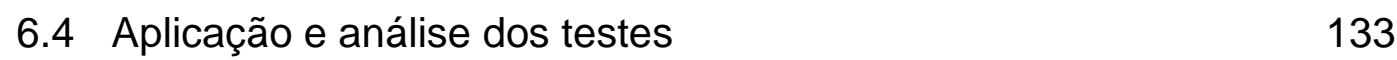

7 Considerações finais 141

8 Referências Bibliográficas $\quad 167$

9 Glossário 172

$\begin{array}{lll}9.1 \text { Definições } & 172\end{array}$

9.2 Conceitos 173

$\begin{array}{ll}9.3 \text { Incapacidades } & 173\end{array}$

$\begin{array}{lll}9.4 & \text { Dispositivos } & 174\end{array}$

$\begin{array}{lll}9.5 & \text { Exigências normativas e legais } & 175\end{array}$

10 Anexos 176

10.1 Modelo de questionário 176

10.2 Modelo do Termo de Consentimento Livre e Esclarecido 178 10.3 Questionários Aplicados e Termos de Consentimento Assinados 179 


\section{Lista de figuras}

Figura 1 - Nicolelis e o exoesqueleto 22

Figura 2 - Exoesqueleto em teste 22

Figura 3 - Juliano Pinto com exoesqueleto na Copa 2014

Figura 4 - Ortostática sentada 23

Figura 5 - Ortostática em pé 23

Figura 6 - Ortostática em uso 23

Figura 7 - Ortostática: usuário em pé 24

Figura 8 - Trigonal Tower, 1962 - Snelson 24

Figura 9 - Early X-Piece, 1948 - Snelson 24

Figura 10 - Cúpula geodésica, 1967 - Fuller 25

Figura 11 - Tensegrity esférico, 1927 - Fuller 25

Figura 12 - Escultura Needle Tower, 1968 - Snelson 25

Figura 13 - Arquitetura Kurilpa Bridge - Brisbane, Queensland, Austrália 26

Figura 14 - Modelo tensegrity - Ripper / LILD 26

Figura 15 - Andadores - LILD e CVI 26

Figura 16 - Aplicação tensegrity em produto - LILD 26

Figura 17 - Cadeira de rodas de estrutura rígida 127

Figura 18 - Cadeira de rodas de estrutura rígida 2

Figura 19 - Cadeira de rodas de estrutura rígida 3

Figura 20 - Cadeira de rodas ortostática - Projeto do autor da tese $\quad 30$

Figura 21 - Cadeira em aço dobrável 32

Figura 22 - Cadeira Ultraleve em alumínio 32

Figura 23 - Cadeira em fibra de carbono para competição 32

Figura 24 - Cadeira de rodas elétrica 32

Figura 25 - Projeto Excludente 33

Figura 26 - Ergodesign 33

Figura 27 - Exemplo de avaliação dimensional preliminar 34

Figura 28 - Cadeiras de rodas expostas para comercialização em lojas 35

Figura 29 - Processo Usual X Processo Ideal 36

Figura 30 - Relação entre a base na posição ortostática e sentada $\quad 37$ 
Figura 31 - Evolução postural do homem ao cadeirante

Figura 32 - Esforços na postura ortostática - Kapandji, 2000

Figura 33 - Esforços na pélvis na postura sentada Kapandji, $2000 \quad 39$

Figura 34 - Postura sentada - Kapandji, 2000

Figura 35 - Pressões intradiscais nas diversas posturas 41

Figura 36 - Postura correta na cadeira de rodas 42

Figura 37 - Equilíbrio ortostático é anteriozado - equilíbrio dinâmico 42

Figura 38 - Postura cifosada com quadril retro vertido 43

Figura 39 - Postura cifosada com quadril na posição vertical 43

Figura 40 - Postura lordosa com anteroversão excessiva 44

Figura 41 - Fixação da Pélvis $\quad 45$

Figura 42 - Inclinação do tronco na horizontalização do olhar 46

Figura 43 - Assento estreito 38

Figura 44 - 3 tipos de instabilidade de tronco 38

Figura 45 - Assento largo $\quad 49$

Figura 46 - Profundidade ideal do assento 49

Figura 47 - Assento comprido $\quad 49$

Figura 48 - Assento curto $\quad 49$

Figura 49 - Facilidades na Transferência $\quad 50$

Figura 50 - Instabilidade do Assento $\quad 50$

Figura 51 - Coluna humana $\quad 51$

Figura 52 - Altura do encosto 51

Figura 53 - Pressão nos pés 52

Figura 54 - Altura do pedal 52

Figura 55 - Early X-Piece, 1948 - Snelson 56

Figura 56 - Escultura Tensegrity - Snelson - detalhe 56

Figura 57 - Estrutura molecular 56

Figura 58 - Estrutura molecular epitelial $\quad 57$

Figura 59 - Cúpula geodésica, 1967 - Fuller 57

Figura 60 - Tensegrity esférico, 1927 - Fuller 57

Figura 61 - Teto Estaiado do Estádio Olímpico de Munique - Frei Otto e Gunther Behnisch 57

Figura 62 - Ponte Estaiada Transcarioca - Rio de Janeiro 57

Figura 63 - Combinações de células tensegrity - Snelson 58 
Figura 64 - Combinações de base triangular - Snelson 58

Figura 65 - Montagem de Tensegrity Eliptico - Snelson 59

Figura 66 - Detalhe da montagem Eliptica - Snelson 59

Figura 67 - Formas diferenciadas de Tensegrity - Snelson 59

Figura 68 - Demonstração da maleabilidade do modelo tensegrity esférico $\quad 60$

Figura 69 - Escultura tensegrity maleável- Snelson 60

Figura 70 - Modelo tensegrity Ripper /LILD adaptado

para a Cadeira de Rodas Tensegrity 60

Figura 71 - Modelo tensegrity Ripper /LILD 60

Figura 72 - Forças no modelo tensegrity 61

Figura 73 - Modelo tensegrity tetraedro simulando a curvatura de uma coluna sineticamente combinada 62

Figura 74 - Coluna vertebral em tensegrity -

Doutorado Geórgia Victor PUC-Rio 2008

Figura 75 - Detalhe Coluna vertebral tensegrity -

Doutorado Geórgia Victor PUC-Rio 2008

Figura 76 - Tetraedro formado por dois triângulos - Baldwin 63

Figura 77 - Modelo tensegrity - Ripper / LILD 64

Figura 78 - Vortex III Snelson 64

Figura 79 - Esboço Vortex III Snelson 64

Figura 80 - Detalhe Vortex III Snelson 64

Figura 81 - Patente tensegrity - Snelson 65

Figura 82 - Kenneth Snelson 66

Figura 83 - Escultura X-Tend - Snelson 66

Figura 84 - Escultura Eight Up - Snelson 66

Figura 85 - Escultura Eight Up - Snelson $\quad 67$

Figura 86 - Escultura QuinTet Row - Snelson 67

Figura 87 - Escultura V-X - Snelson 67

Figura 88 - Escultura Easy Landing - Snelson 68

Figura 89 - Torre EC Column - Detalhe 68

Figura 90 - Torre EC Column - Snelson 68

Figura 91 - Escultura Needle Tower - Detalhe 69

Figura 92 - Escultura Needle Tower - Snelson 69 
Figura 93 - Kurilpa Bridge - Brisbane, Queensland, Austrália

Figura 94 - Frei Otto

Figura 95 - Kurilpa Bridge - detalhe $1 \quad 70$

Figura 96 - Kurilpa Bridge - detalhe $2 \quad 70$

Figura 97 - Pavilhão Alemão em Montreal - Detalhe 71

Figura 98 - Pavilhão Alemão Exposição Montreal - Otto 1967

Figura 99 - Estádio Olímpico de Munique - Interno - Otto 1972

Figura 100 - Estádio Olímpico de Munique - Otto 1972

Figura 101 - Aviário do Zoo de Munique 73

Figura 102 - Tenda Coração - Detalhe externo 73

Figura 103 - Tenda Coração no Tuwaiq Palace em Riyadh - Otto 73

Figura 104 - Tenda Coração - Detalhe 2

Figura 105 - Guarda-sol $\quad 73$

Figura 106 - Guarda-sol show Pink Floyd EUA - Otto 1977

Figura 107 - Modelos das estruturas - Otto 74

Figura 108 - Bicicleta tensegrity idealizada por Flavio Deslandes no LILD/PUC-Rio 75

Figura 109 - Bicicleta tensegrity Maquete $\quad 75$

Figura 110 - Bicicleta tensegrity - Detalhe 75

Figura 111 - Bicicleta tensegrity - Inout Predescu - Romenia 76

Figura 112 - Bicicleta tensegrity Inout Predescu - Detalhe 76

Figura 113 - Bicicleta tensegrity Inout Predescu - Detalhe 2

Figura 104 - Mesa de café tensegrity Theodore Waddell 76

Figura 115 - Mesa tensegrity Theodore Waddell - Detalhe $2 \quad 77$

Figura 116 - Mesa tensegrity Theodore Waddell - Detalhe $3 \quad 77$

Figura 117 - Mesa tensegrity de Theodore Waddell 77

Figura 118 - Mesa e cadeira X-tense de Konstantin Achkov 78

Figura 119 - Mesa X-tense de Konstantin Achkov - Detalhe 78

Figura 120 - Mesa e cadeira V-star de Konstantin Achkov 78

Figura 121 - Cadeira X-tense de Konstantin Achkov 78

Figura 122 - Mesa V-star de Konstantin Achkov - Detalhe 78

Figura 123 - Mesa V-star de Konstantin Achkov - Detalhe $2 \quad 78$

Figura 124 - Mesa Rotor de Konstantin Achkov - Modelo 1

Figura 125 - Mesa Rotor de Konstantin Achkov - Modelo 2 
Figura 126 - Mesa Rotor Modelo 2 - Detalhe 79

Figura 127 - Mesa Rotor Modelo 2 - Detalhe 2

Figura 128 - Mesa Rotor Modelo 2 - Detalhe 3

Figura 129 - Pipa plana de crianças $\quad 80$

Figura 130 - Estrutura de pipas duplicadas e paralelas $\quad 80$

Figura 131 - Estrutura de pipas inseridas num paralelepípedo 81

Figura 132 - Estrutura tensegrity com haste transversal 81

Figura 133 - Estrutura com haste transversal cabeada 81

Figura 134 - Módulo tensegrity prof. Ripper com medidas 82

Figura 135 - Módulo tensegrity original do prof. Ripper 82

Figura 136 - Estrutura tensegrity do designer Mário Seixas 82

Figura 137 - Barraca de estrutura tensegrity - designer Mário Seixas 83

Figura 138 - Barraca de estrutura tensegrity

em uso - designer Mário Seixas 83

Figura 139 - Módulos tensegrity desenvolvidos pelo Lild 83

Figura 140 - Conjunto de uso $\quad 84$

Figura 141 - Conjunto de uso e Rodas sem estrutura (quadro) 84

Figura 142 - Desenho técnico do conjunto deuso e rodas

no padrão dimensional $\quad 85$

Figura 143 - Módulo tensegrity girado com hastes paralelas ao chão coincidentes com as rodas dianteiras

Figura 144 - Coincidência da posição das rodas

dianteiras com as hastes paralelas

Figura 145 - Módulo tensegrity com coincidência

das varas de bambu verticais com o conjunto de uso

87

Figura 146 - Coincidência da posição da estrutura

do assento com as hastes verticais

Figura 147 - Módulo tensegrity com encaixe da

haste de bambu com as rodas traseiras

Figura 148 - Coincidência da posição das rodas traseiras com a haste transversal

Figura 149 - Conjunto de uso e módulo tensegrity posicionados $\quad 88$

Figura 150 - Módulo tensegrity ajustado ao Conjunto de uso 89

Figura 151- Tensegrity com haste faceada a roda traseira 89 
Figura 152 - Ajuste das hastes verticais para assumir simetria

Figura 153 - Conjunto de uso e tensegrity com conexões e cabos

Figura 154 - Ajuste das hastes verticais para assumir simetria

Figura 155 - Design do protótipo da cadeira de rodas de estrutura tensegrity

Figura 156 - Projetos das conexões com as presilhas do cabo de aço

Figura 157 - Estrutura tensegrity com as conexões nas extremidades das hastes de bambu e indicação de encaixe ao conjunto de uso

Figura 158 - Conexões nas extremidades das hastes de bambu que nãopossuem encaixe ao conjunto de uso e presilhas do cabo de aço na base e borda

Figura 159 - Encaixe superior da estrutura tensegrity com o conjunto de uso com presilhas do cabo de aço na borda

Figura 160 - Encaixe da estrutura tensegrity com o eixo rodas

Figura 161 - Acoplamentos da estrutura tensegrity, rodas dianteiras; parte inferior do conjunto de uso e presilhas do cabo de aço

Figura 162 - Projeto das presilhas do cabo de aço na conexão

Figura 163 - Ponto de convergência da força dos

cabos de aço dentro da conexão

Figura 164 - Kit do cabeamento esticador,

cabo de aço, travas e mosquetão

Figura 165 - Ponto de convergência da força dos cabos de aço fora da conexão

Figura 166 - Conexão com embuchamento de linha grossa de algodão 95 Figura 167 - Comparação do projeto e do modelo de testes da Cadeira de rodas tensegrity 96 Figura 168 - Projeto do conjunto de uso $\quad 97$

Figura 169 - Conjunto de uso do modelo de teste em duralumínio $\quad 97$

Figura 170 - Presilhas do cabo de aço soldadas na conexão 98

Figura 171 - Tubos cilíndricos de aço-carbono das conexões 98

Figura 172 - Pisos laminados de bambu 99

Figura 173 - Variedades de bambu 99

Figura 174 - Ambiente de bambu 99 
Figura 175 - Touceira de bambu sem limpeza e aproveitamento

Figura 176 - Limpeza da touceira de bambu

Figura 177 - Mudas de bambu

Figura 178 - Caules jovens na touceira limpa

Figura 179 - Touceira limpa

Figura 180 - Bambu - mirim ou Phyllostachys aurea

102

Figura 181 - Lagarta atacando o interior

do Bambu Phyllostachys aurea

102

Figura 182 - Divisões e nós do bambu Phyllostachys aurea 102

Figura 183 - Variação diametral

103

Figura 184 - Corte na borda do Bambu

103

Figura 185 - Segmento de bambu com camada protetora superficial 104

Figura 186 - Pesquisa Lucas Ripper - Estrutura curvada 104

Figura 187 - Bambu cru ou natural sem tratamento 105

Figura 188 - Tratamento superficial com resina

(esquerda) e betume (direita)

105

Figura 189 - Tratamento superficial: Doutorado de Arisio Rabin, PUC-Rio 2015

Figura 190 - Tintas naturais de tratamento superficial: Doutorado de Arisio Rabin, PUC-Rio 2015

Figura 191 - Detalhe: Doutorado de Arisio Rabin, PUC-Rio 2015

Figura 192 - Padrões de design e cores nos bambus: Doutorado de Arisio Rabin, PUC-Rio 2015

Figura 193 - Gabarito para fabricação de cadeira estudantil 108

Figura 194 - Gabarito para fabricação de cadeira 108

Figura 195 - Gabarito para fabricação de estrutura metálica 108

Figura 196 - Embraer $196 \quad 109$

Figura 197 - Gabarito do avião Embraer 190

Figura 198 - Gabarito do avião Embraer 190 - 2

Figura 199 - Linha de montagem automotiva 109

Figura 200 - Oficina UFRJ 110

Figura 201 - Estrutura rígida e móvel da cadeira de rodas ortostática 110

Figura 202 - Ortostática: usuário em pé 110

Figura 203 - Vista Lateral do conjunto de uso 
aproveitado da Cadeira Ortostática

Figura 204 - Conjunto de uso virtual

Figura 205 - Conjunto de uso da cadeira de rodas ortostática

Figura 206 - Desenho técnico do gabarito para

o suporte do conjunto de uso

Figura 207 - Ortostática: chassi com o sistema de elevação 112

Figura 208 - Conjunto de uso com estrutura tensegrity 112

Figura 209 - Gabarito: Criação da base de madeira 113

Figura 210 - Gabarito: Medições para confecção da base 113

Figura 211 - Gabarito: Base esquadrinhada 113

Figura 212 - Gabarito: União da base com cantoneiras 113

Figura 213 - Gabarito: Apoios dianteiros do conjunto de uso 114

Figura 214 - Gabarito: Encaixe côncavo dos tubos 114

Figura 215 - Gabarito: Amarração transversal das

hastes de suporte dianteiras $\quad 114$

Figura 216 - Gabarito: Parte de sustentação das rodas traseiras 114

Figura 217 - Gabarito: Apoios traseiros dos tubos do assento 114

Figura 218 - Gabarito com suporte do conjunto de uso montado $\quad 115$

Figura 219 - Estrutura tensegrity com conjunto de uso 115

Figura 220 - Imagem virtual da estrutura tensegrity 116

Figura 221 - Vista frontal - cadeira de rodas tensegrity 116

Figura 222 - Desenho técnico cadeira de rodastensegrity - vista lateral 116

Figura 223 - Estrutura tensegrity com conjunto de uso 117

Figura 224 - Imagem virtual da estrutura tensegrity 117

Figura 225 - Haste horizontal $\quad 117$

Figura 226 - Haste transversal (indicado em verde) e

suporte (indicado em branco) 117

Figura 227 - Hastes verticais $\quad 118$

Figura 228 - Conexão estrutura tensegrity com conjunto de uso 118

Figura 229 - Gabarito pronto com as cinco hastes posicionadas 118

Figura 230 - Gabarito montagem 118

Figura 231 - Gabarito montagem $2 \quad 118$

Figura 232 - Corte dos tubos de 1'1/4" 119

Figura 233 - Tubos cortados 119 
Figura 234 - Tubos conexão com tampas soldadas

Figura 235 - Conexão dos cabos

Figura 236 - Imagem virtual da estrutura tensegrity

Figura 237 - Conexão da roda traseira

Figura 238 - Conexão da roda dianteira

Figura 239 - Acabamento das hastes horizontais

com encaixe do conjunto de uso

120

Figura 240 - Acabamento das hastes horizontais finalizado

Figura 243 - Acabamento das extremidades das hastes verticais

Figura 244 - Distanciador das hastes verticais

Figura 245 - Distanciador das hastes horizontais

Figura 246 - Gabarito montado com hastes e conexões de metal

Figura 247 - Cadeira de rodas tensegrity

Figura 248 - Cabo de aço laçado com esticador

Figura 249 - Mosquetão

Figura 250 - Esticador de cabo girado no sentido horário

Figura 251 - Esticador de cabo girado no sentido anti-horário

Figura 252 - Presilha do cabo de aço

Figura 253 - Cabo de aço travado 124

Figura 254 - Sistema de passar cabo 124

Figura 255 - Imagem virtual da estrutura tensegrity 125

Figura 256 - Conjunto do cabeamento hastes horizontais 125

Figura 257 - Conjunto do cabeamento hastes verticais 125

Figura 258 - Destaque conjunto cabeamento ligações obliquas $\quad 125$

Figura 259 - Parte traseira montada e cabeada $\quad 125$

Figura 260 - Imagem virtual da estrutura tensegrity 126

Figura 261 - Cadeira de Rodas Tensegrity em exposição na PUC-Rio 126

Figura 262 - Orientador da pesquisa testando a cadeira tensegrity $\quad 126$

Figura 263 - Modelo de testes da cadeira de rodas tensegrity e cadeira de rodas AVD

Figura 264 - Renata Vargas a primeira usuária dos testes da cadeira tensegrity

Figura 265 - Reunião do grupo multidisciplinar no CVI/PUC-Rio $\quad 135$

Figura 266 - Renata Vargas a na reunião no CVI/PUC-Rio 
Figura 267 - Willian de Sousa a segunda pessoa

dos testes com a cadeira tensegrity

Figura 268 - Willian de Sousa realizando

atividade na cadeira tensegrity

138

Figura 269 - Willian de Sousa utilizando a cadeira tensegrity $\quad 139$

Figura 270 - Willian de Sousa nos testes com a cadeira tensegrity 139

Figura 271 - Dr. José Carlos Moraes nos testes

e análises da cadeira tensegrity 140

Figura 272 - Partes alteradas no projeto da cadeira tensegrity 142

Figura 273 - Encurtamento da estrutura da cadeira tensegrity 144

Figura 274 - Variação de altura da cadeira tensegrity 144

Figura 275 - Chapa com uma furação em cada lado 144

Figura 276 - Chapa com três furações para regulagem 145

Figura 277 - Encaixe de 3 estágios na parte frontal 145

Figura 278 - Sistema de Engate com detalhe do encaixe no eixo 145

Figura 279 - Trava do Sistema de Encaixe 146

Figura 280 - Afastador das rodas dianteiras 147

Figura 281 - Barra estabilizadora $\quad 147$

Figura 282 - Rebaixamento do apoio dos pés $\quad 147$

Figura 283 - Assento rígido na cadeira tensegrity 147

Figura 284 - Angulação das presilhas dos cabos de aço 148

Figura 285 - Bambu fosco claro e escuro 149

Figura 286 - Cadeira de rodas tensegrity em bambu fosco 149

Figura 287 - Cadeira de rodas tensegrity em bambu brilhante $\quad 150$

Figura 288 - Cadeira de rodas tensegrity em bambu escuro brilhante 151

Figura 289 - Bambu com padrões e cores - Doutorado Arisio 152

Figura 290 - Acabamento superficial do bambu - Doutorado Arisio 152

Figura 291 - Cadeira de rodas tensegrity em bambu

pintado de preto com padrão de listras coloridas 153

Figura 292 - Detalhe do bambu pintado de preto com listras coloridas 153

Figura 293 - Cadeira de rodas tensegrity em bambu

pintado de azul com padrão de listras coloridas

Figura 294 - Detalhe do bambu pintado de azul com listras coloridas 154

Figura 295 - Cadeira de rodas tensegrity em bambu 
pintado de vermelho com padrão esfumaçado

Figura 296 - Detalhe do bambu pintado de vermelho com esfumaçado preto 155

Figura 297 - Cadeira de rodas tensegrity em bambu amarelo 156

Figura 298 - Cadeira de rodas tensegrity em fibra de carbono clara 157

Figura 299 - Cadeira de rodas tensegrity em fibra de carbono escura 158 Figura 300 - Cadeira de rodas tensegrity híbrida

de metal e bambu claro

Figura 301 - Cadeira de rodas tensegrity híbrida

de metal bambu escuro

Figura 302 - Cadeira de rodas tensegrity híbrida

metal escuro com bambu escuro

Figura 303 - Detalhe do conjunto das conexões com cabeamento 162

Figura 304 - Touceira de bambu

162

Figura 305 - Alongamento das presilhas

163

Figura 306 - Perspectiva das conexões

163

Figura 307 - Alteração da direção das presilhas

163

Figura 308 - Facilidade de enlaçamento do cabo de aço 163

Figura 309 - Exemplo de produção complexa por impressão 3D 164

Figura 310 - Imagens das peças em aço inox sem soldas 165

Figura 311 - Conexão nova cabeada e montada na cadeira 166 


\section{Lista de tabelas}

Tabela 1 - Quadro de avaliação

Tabela 2 - Relação resistência à tração $X$ peso específico

Fonte: Ghavami (1992)

Tabela 3 - Relação energia de produção por unidade de tensão Fonte: Ghavami (1992) 